necessary for spindle assembly. The answer is an unequivocal 'yes'.

Perhaps, then, Rae1 has different functions - mRNA export or spindle assembly - depending on the proteins with which it associates during the different phases of the cell cycle. During spindle assembly, its function might be purely to function as a 'scaffold', bringing in necessary proteins. But what about RNA? It doesn't seem to be required for new protein synthesis during spindle assembly. But it might, the authors speculate, help to maintain the integrity of the Rael-bound protein complex. Or it might have a more active role, influencing the activity of these proteins.

Amanda Tromans, Senior Editor, Nature 6) References and links ORIGINAL RESEARCH PAPER Blower, M. D., Nachury, M., Heald, R. \& Weis, K. A Rae1containing ribonucleoprotein complex is required for mitotic spindle assembly. Cell 121, 223-234 (2005)

WEB SITES

Rebecca Heald's laboratory:

http://mcb.berkeley.edu/labs/heald/

Karsten Weis' laboratory:

http://mcb.berkeley.edu/faculty/CDB/weisk.htm

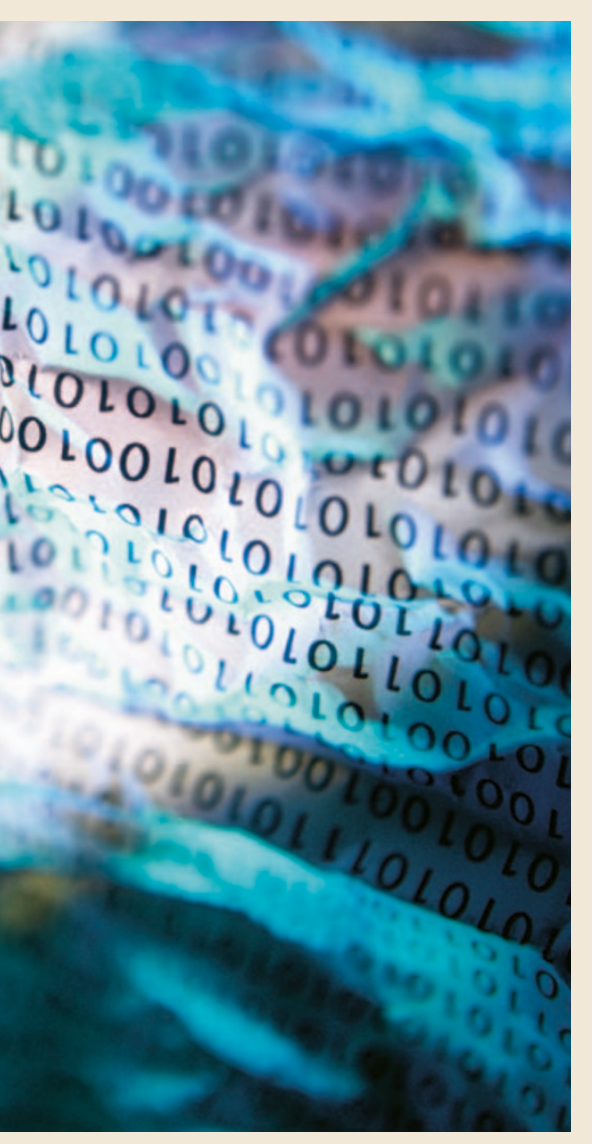

\section{POST-TRANSLATIONAL MODIFICATION}

\section{Jack of all trades}

Think of methyltransferases, and histone modification and epigenetic regulation probably spring to mind. Indeed, the Polycomb group protein Ezh2 does regulate histone $\mathrm{H} 3$ methylation on lysine residues, which is important for its key regulatory role in development. But Su et al., reporting in Cell, found that Ezh2 also liaises with cytosolic proteins.

The first clue to the extracurricular activity of Ezh2 came with the observation that it localizes in the cytosol of several cell types, including thymocytes, peripheral T cells and mouse embryonic fibroblasts. Activation of the T-cell antigen receptor (TCR) in T cells increased the amount of cytosolic Ezh2.

Nuclear Ezh2 requires Suz12 and Eed to exert its methyltransferase activity. But these proteins also co-immunoprecipitated with Ezh2 when the nuclear localization signal of Ezh2 was deleted (Ezh2 $\Delta$ NLS), and the complex, when incubated with histone $\mathrm{H} 3$, showed methyltransferase activity (although histone $\mathrm{H} 3$ is obviously not a cytosolic substrate).

Ezh2 has previously been reported to associate with the guanine nucleotide-exchange factor Vav1, an interaction that the authors also observed in Jurkat cells. Suz12 and Eed also co-precipitated with Vav1, implying that a functional cytosolic Ezh2 methyltransferase complex did exist. Could Ezh2 regulate Vav1, or vice versa? As Vav regulates TCR-mediated actin polymerization, Su et al. studied the former possibility, using Ezh2-deficient peripheral T cells.

Although overall histone H3 Lys27 methylation was unaffected, actin polymerization could not be induced in response to TCR activation in Ezh2-deficient T cells, and filamentous (F)-actin was not recruited to the interface between $T$ cells and antigen-presenting cells. In wild-type fibroblasts, platelet-derived growth factor (PDGF) induced membrane ruffles and, when tagged, Ezh2 could be detected at these structures. Membrane ruffles couldn't be induced in Ezh2-deficient mouse embryonic fibroblasts (MEFs) in response to PDGF until wild-type or Ezh2 $\Delta$ NLS was expressed. However, a form of Ezh2 that lacked methyltransferase activity could not rescue membrane ruffling.
Actin polymerization requires the Rho GTPase Cdc42, which activates the actin-nucleating Arp2/3 complex through Wiskott-Aldrich syndrome protein. Activation of the TCR increased the levels of active, GTP-bound Cdc42 in wild-type, but not Ezh2-deficient, T cells. Tyrosine phosphorylation of the TCR and its substrates was normal in Ezh2-deficient $\mathrm{T}$ cells, as was phosphorylation of a key tyrosine residue in Vav1. So what lies immediately upstream and downstream of Ezh2 in this response is still elusive. But because expressing a constitutively active form of $\mathrm{Cdc} 42$ in Ezh2-deficient $\mathrm{T}$ cells and fibroblasts restored the response of these cells to TCR activation and PDGF, respectively, cytosolic Ezh2 seems to function upstream of Vav1 and Cdc42.

Not only did a lack of Ezh2 inhibit actin polymerization in response to TCR, it also inhibited proliferation in these cells. Similarly, Ezh2-deficient fibroblasts showed a reduced proliferative response to PDGF (but not to serum, which contains many growth factors). Wild-type and cytosolic Ezh2, but not a cytosolic methyltransferase-inactive form of Ezh2, could rescue the proliferative response. Finally, the authors discovered a role for Ezh2 in T-cell development in the thymus, which is probably mediated by a signalling function in pre-TCRmediated thymocyte development. Not bad for a protein that was thought 'simply' to control gene activity through histone methylation; the findings also extend the role of protein lysine methylation to the regulation of proliferation and actin polymerization in the cytosol.

Katrin Bussell

(2) References and links

ORIGiNAL Research PAPER Su, I.-H. et al. Polycomb group protein Ezh2 controls actin polymerization and cell signalling. Cell 121, 425-436 (2005) 